\title{
Síndrome agudo respiratorio severo: un panorama mundial de la epidemia
}

\author{
Carlos Franco-Paredes, MC, MPH, (1) Pablo Kuri-Morales, MC, M en C, ${ }^{(2)}$ Carlos Alvarez-Lucas, CD, MSS, ${ }^{(2)}$ \\ Ethel Palacios-Zavala, MC, (2) Margarita Nava-Frías, MC,(1) Miguel Betancourt-Cravioto, MC, (2) \\ José Ignacio Santos-Preciado, BSc, MSc, MD, (1) Roberto Tapia-Conyer, MC, MSP, MPH. ${ }^{(3)}$
}

\section{Franco-Paredes C, Kuri-Morales P, Alvarez-Lucas C, Palacios-Zavala E, Nava-Frías M, Betancourt-Cravioto $M$, Santos-Preciado JI, Tapia-Conyer R. Síndrome agudo respiratorio severo: un panorama mundial de la epidemia. Salud Publica Mex 2003;45:2II-220. El texto completo en inglés de este artículo está disponible en: http://www.insp.mx/salud/index.html}

\section{Resumen}

A principios de febrero de 2003 la Organización Mundial de la Salud comenzó a recibir reportes de pacientes con un síndrome caracterizado por neumonía atípica, con rápida progresión hacia insuficiencia respiratoria sin una causa identificada. Los casos aparentemente se iniciaron en el sur de China y se han diseminado a otras regiones en Asia, Europa, Sudáfrica, Norte América y Sur América. La causa de este síndrome es una nueva variedad de Coronavirus, aislado en secreciones respiratorias y en otras. El síndrome ha sido definido en inglés como SARS (Severe acute respiratory syndrome) por la Organización Mundial de la Salud y se caracteriza por un periodo de incubación de I a I0 días (promedio de cinco días), una fase febril prodrómica que aparece entre los días I a 3. Posteriormente, aparecen síntomas respiratorios como tos, disnea, y signos como hipoxemia, que en 10 a $40 \%$ de los casos requieren de ventilación mecánica. La tasa de letalidad ha variado de $3 \%$ hasta $16 \%$. Los hallazgos de laboratorio incluyen trombocitopenia, leucopenia, elevación de creatinin-fosfokinasa, y, en ocasiones, de transaminasas hepáticas y deshidrogenasa láctica. El tratamiento incluye medidas de apoyo; la utilización empírica del antiviral ribavirina es controvertida, debido a que hasta el momento no existe un tratamiento específico. Se recomienda el aislamiento respiratorio de los pacientes, la utilización de máscaras protectoras y el lavado estricto de manos como
Franco-Paredes C, Kuri-Morales P, Alvarez-Lucas C, Palacios-Zavala E, Nava-Frías M, Betancourt-Cravioto M, Santos-Preciado JI, Tapia-Conyer R.

Severe Acute Respiratory Syndrome:

A Global View of the Epidemic.

Salud Publica Mex 2003;45:2 I I-220.

The English version of this paper

is available at: http://www.insp.mx/salud/index.html

\section{Abstract}

In early February 2003, the World Health Organization (WHO) began receiving reports of patients with a syndrome characterized by an atypical pneumonia with rapid progression to respiratory failure without an identified cause despite extensive diagnostic workups. Most of these reports pointed out that the outbreak started in Southern China, specifically in the Guandong Province. The initial outbreak in South East Asia has already spread to other Regions in Asia, Europe, North and South America, and South Africa. Many of these cases can be linked through chains of transmission to an index case from the Guandong Province who visited Hong Kong. Although the exact mode of transmission has not been clearly established, the etiology of this syndrome has already been identified. A novel Coronavirus has been identified by electron microscopy and molecular assays in multiple laboratories from respiratory specimens throughout the world. The syndrome has been defined as SARS (Severe Acute Respiratory Syndrome) by WHO, and is characterized by an incubation period between I and 10 days (average 5 days) and by a febrile phase that usually lasts approximately 3 days. During the respiratory phase that begins around day 3, patients start developing a dry cough, shortness of breath and hypoxemia. Mechanical ventilatory support is required in about 10 to $40 \%$ of cases and the case-fatality rate ranges between 3 and $16 \%$. The labo-

(I) Centro Nacional para la Salud de la Infancia y Adolescencia, Secretaría de Salud. México, DF, México.

(2) Dirección General de Epidemiología, Secretaría de Salud. México, DF, México.

(3) Subsecretaría de Prevención y Protección a la Salud, Secretaría de Salud. México, DF, México.

Fecha de recibido: 8 de mayo de 2003 - Fecha de aprobado: 2 de junio de 2003

Solicitud de sobretiros: José Ignacio Santos Preciado, Director General, Centro Nacional para la Salud de la Infancia y Adolescencia y Secretario Técnico del Consejo Nacional de Vacunación. Francisco P. Miranda 177 2do. Piso, colonia Merced Gómez, 01600 México, DF.

Correo electrónico: jisantos@supernet.com.mx 
principales medidas de prevención. Desde el inicio de esta epidemia México estableció un sistema de vigilancia, así como recomendaciones al personal de salud para la identificación, prevención de casos secundarios y manejo clínico de casos sospechosos. El texto completo en inglés de este artículo está disponible en: http://www.insp.mx/salud/index.html

Palabras clave: síndrome agudo respiratorio severo; insuficiencia respiratoria; Coronavirus; neumonía atípica ratory findings in SARS cases include leukopenia, thrombocytopenia, and a rise in transaminases and lactic dehydrogenase levels.Treatment of SARS includes supportive measures and the empiric use of ribavirin. Respiratory isolation, use of respiratory masks, and compulsory hand hygiene constitute the principal preventive measures. The confirmation of a case can be performed at reference laboratories by serologic and molecular assays. From the onset of this epidemic Mexico established a surveillance system as well as clinical guidelines and recommendations for the identification, prevention of secondary spread, and medical management of suspicious and probable cases by health care personnel. The English version of this paper is available at: http:// www.insp.mx/salud/index.html

Key words: severe acute respiratory syndrome; respiratory insufficiency; Coronavirus; pneumonia, atypical
E 1 síndrome agudo respiratorio severo o severe acute respiratory syndrome (SARS), por sus siglas en inglés, es una infección emergente en la cual se ha identificado a una nueva variedad del Coronavirus como el agente etiológico. Este síndrome se manifiesta clínicamente desde un síndrome febril asociado a síntomas respiratorios leves, hasta una neumonía rápidamente progresiva hacia insuficiencia respiratoria, asociada a una tasa de letalidad de 3 a 4\%. Esta epidemia se considera que se originó en la provincia de Guandong, en el sur de China, la cual se ha diseminado hasta el momento a otros países en Asia, y hacia otras regiones como Australia, Norte América, Sur América, Sur Africa y Europa. ${ }^{1-5}$

A pesar de que los primeros reportes, que recibió la Organización Mundial de la Salud (OMS), de este síndrome ocurrieron a principios de febrero de 2003, se sabe que los primeros casos se dieron a finales de noviembre en la ciudad de Guangzhou, provincia de Guandong, China. El gobierno chino decidió no reportar estos casos a tiempo, por lo cual se considera que la epidemia se ha expandido a otras regiones dentro del territorio chino, así como a otros países. La OMS, que rara vez confronta a sus países miembros, reclamó oficialmente al gobierno chino por el ocultamiento de los casos. Debido a estas acciones, el ministro de salud de China y el gobernador de Beijing fueron destituidos de sus puestos a principios de abril. Asimismo, el gobierno de Beijing, ante el reclamo de la OMS, ha permitido la colaboración de miembros de ésta en hospitales de Guangzhou y de Beijing, en donde, inclusive, algunos han tenido que cerrar su acceso al público. ${ }^{6}$ El costo económico y social del SARS ha sido muy elevado; se calcula que el crecimiento económico de China y de la Región Asiática puede disminuir hasta en $50 \%$ en este año. ${ }^{6,7}$ Las restricciones para viajar a la Región, el establecimiento de cuarentenas de aislamiento y el cierre de edificios públicos, incluyendo hospitales, se han incrementado en las últimas semanas en China y Hong Kong. ${ }^{6-8}$

Casos del SARS y notas de actualización sobre la epidemia en el mundo son reportados diariamente. ${ }^{4,9,10}$ Más aun, el número de casos en China, Hong Kong, Taiwán, Canadá y Estados Unidos de América se incrementa día con día. ${ }^{2}$ Hasta finales de mayo, más de 8295 casos, en más de 20 países, con 750 muertes descritas entre el 1 de febrero y el 30 de mayo de 2003, han sido reportados a la OMS. En este momento no se puede predecir el número de casos que ocurrirán, sin embargo, se espera que el número aumente de manera importante y se disemine a otros paises. ${ }^{2,4}$ Es probable que la transmisión del SARS evolucione para imitar las pandemias de influenza, para lo cual la ausencia de vacuna disponible hasta el momento, y de caracterización epidemiológica precisa, representan enormes obstáculos para un adecuado control. Por lo anterior, resulta como prioridad el establecimiento de un plan que contenga medidas de control para la prevención e identificación temprana de casos del SARS en México y América Latina. Aunado a las medidas de control epidemiológico es fundamental proporcionar información pertinente al personal de salud para la identificación clínica de casos, la prevención de los casos secundarios, y el tratamiento de los sospechosos y probables, con aislamiento respiratorio y tratamiento médico de apoyo. 


\section{Epidemiología}

\section{Caracterización mundial dela epidemia}

El origen de esta epidemia aparentemente comienza en la provincia de Guandong, en el sur de China. Ha habido casos en China, Hong Kong, Taiwán, Vietnam, Singapur, Canadá y en otros países señalados en el cuadro I. ${ }^{1-5}$

\section{Etiología delSARS}

El análisis inicial de los brotes de casos del SARS descritos en Canadá, Estados Unidos de América (EUA) y en Hong Kong ${ }^{11-14}$ ha sugerido la posibilidad de una variedad nueva de Coronavirus y una nueva variedad de Metaneumovirus humano como posibles agentes etiológicos. La posible asociación de una nueva variedad de Metaneumovirus humano se sugirió por la identificación del virus en cinco pacientes de los casos iniciales descritos en Canadá, y en algunos pacientes con el SARS en Hong Kong. ${ }^{11,12}$ La similitud del SARS con manifestaciones clínicas ya descritas en la literatura, ocasionadas por el Metaneumovirus humano, sugirió inicialmente una asociación causal entre el SARS y este virus. ${ }^{15,16}$ Sin embargo, el aislamiento del Coronavirus en múltiples laboratorios alrededor del mundo, utilizando las mismas técnicas moleculares en muestras clínicas respiratorias, apuntaba principalmente a este virus como el posible agente etiológico. ${ }^{8,17-19}$ Con el cumplimiento de los postulados de Koch en el modelo animal de primate no humano (Macaca fascicularis) se considera al Coronavirus como el agente causal definitivo del SARS. ${ }^{20}$

El 24 de marzo, investigadores del Centro para el Control de las Enfermedades Infecciosas de Atlanta (CDC) y en Hong Kong 3,5,11,15 detectaron, por microscopía electrónica, la presencia del mismo Coronavirus encontrado en Canadá en las secreciones respiratorias de pacientes con el SARS. El análisis molecular del virus fue comparado con otras secuencias características de previas variedades de Coronavirus, demostrando que se trata de una nueva variedad en esta familia de virus no antes descrita. ${ }^{4,5}$

Hasta el momento, 11 laboratorios internacionales que conforman la red de colaboración de la OMS en respuesta a la epidemia, han encontrado evidencia de la nueva variedad de Coronavirus en pacientes con el SARS. La información pertinente a laboratorios incluidos en esta red se encuentra disponible en la siguiente página de Internet http://www.who.int/csr/ sars/testing/en/. El virus ha sido identificado, con resultados similares, en estos laboratorios a través de
Cuadro I

NúMERO DE CASOS DEL SÍNDROME AGUdo RESPIRATORIO SEVERO Y MUERTES ASOCIADAS

A é distribuidos POR PAís DE ACUERDO CON LA OMS, ENTRE EL PERIODO DEL I DE NOVIEMBRE DE 2002

AL 28 DE MAYO DE 2003

Pais

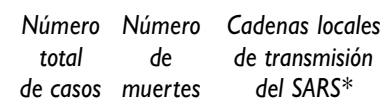

\begin{tabular}{lrrl} 
Australia & 6 & 0 & No \\
\hline Malasia & 5 & 2 & No \\
\hline Brasil & 2 & 0 & No \\
\hline Bulgaria & 1 & 0 & No \\
\hline Canadá & 151 & 26 & Sí \\
\hline China & 5325 & 327 & Sí
\end{tabular}

China, Región Administrativa

\begin{tabular}{lrrl} 
de Hong Kong & $|73|$ & 273 & Sí \\
\hline Colombia & I & 0 & No \\
\hline Kuwait & I & 0 & No
\end{tabular}

\begin{tabular}{lll}
\hline Nueva Zelanda & I & 0 \\
\hline
\end{tabular}

\begin{tabular}{lrrl}
\hline Finlandia & 1 & 0 & No \\
\hline Taiwán & 418 & 52 & Sí \\
\hline Francia & 7 & 0 & No \\
\hline Filipinas & 12 & 2 & No \\
\hline República de Corea & 3 & 0 &
\end{tabular}

\begin{tabular}{lll}
\hline República de Corea & 3 & 0 \\
\hline
\end{tabular}

\begin{tabular}{llll}
\hline España & I & I & \\
\hline Alemania & 9 & 0 & No
\end{tabular}

\begin{tabular}{lrrl}
\hline Alemania & 9 & 0 & No \\
\hline India & 3 & 0 & No \\
\hline Indonesia & 2 & 0 & No \\
\hline Suráfrica & 1 & 1 & No \\
\hline Mongolia & 9 & 0 & No \\
\hline Italia & 9 & 0 & No \\
\hline Irlanda & 1 & 0 & No \\
\hline Japón & 2 & 0 & No \\
\hline Malasia & 5 & 2 & No \\
\hline Rumania & 1 & 0 & No \\
\hline Singapur & 206 & 28 & Si \\
\hline Suiza & 1 & 0 & No \\
\hline Tailandia & 8 & 2 & No \\
\hline Reino Unido & 4 & 0 & No \\
\hline Estados Unidos de América & $66^{\ddagger}$ & 0 & En estudio \\
\hline Vietnam & 63 & 5 & Sí \\
\hline Total & 8295 & 750 &
\end{tabular}

* Las autoridades de salud pública de los países reportan a la OMS si ha habido cadenas locales de transmisión

‡ El Centro para el Control y Prevención de Enfermedades de Atlanta, EUA, utiliza una definición de casos sospechosos que ha incluido un mayor número de ellos; SARS: síndrome agudo respiratorio severo, sin embargo, basándose en la definición de caso sospechoso de la OMS el número de estos casos es únicamente 66.Fuente: Organización Mundial de la Salud, abril de 2003 (Referencia 10) 
diferentes metodologías, incluyendo cultivo de tejidos, microscopía electrónica, inmunofluorescencia indirecta, pruebas de serología con anticuerpos, tecnología de micro-arreglos, reacción de polimerasa en cadena de secuencias genómicas específicas. A través de estudios de inmunofluorescencia se ha documentado la formación de anticuerpos contra la nueva variedad de Coronavirus en sueros de la fase aguda y de la fase de convalecencia, en pacientes con diagnóstico del SARS. ${ }^{8,17,19}$

Peiris y colaboradores demostraron la presencia del Coronavirus en especímenes respiratorios y en suero en 45 de 50 pacientes, provenientes de cinco diferentes brotes del SARS en Hong Kong. Adicionalmente, por cultivos virales se aisló el Coronavirus en dos de estos pacientes. La utilización de un grupo control de 40 pacientes con enfermedades respiratorias, en los cuales no se identificó el ARN viral, y de 200 controles en los cuales se analizó serológicamente la presencia del virus, contribuye en forma importante a establecer la causalidad del Coronavirus con el SARS. ${ }^{19}$

El grupo del CDC ha propuesto la utilización del nombre Coronavirus Urbani-SARS para designar a esta nueva variedad de Coronavirus, en reconocimiento al doctor Carlo Urbani, infectólogo italiano de la OMS, cuyas observaciones de los casos de Vietnam, a fines de febrero y principios de marzo, permitió describir este síndrome y dimensionar la importancia de esta aparente nueva enfermedad. ${ }^{17}$ Lamentablemente, el doctor Urbani se contagió y falleció de la enfermedad que él describió y sobre la cual alertó acerca de su severidad antes de morir.

Hasta el momento se han descrito los genomas virales completos de cinco cepas del Coronavirus, las que varían únicamente en secuencias genómicas que codifican para la proteína de la nucleocápside. De ellas, tres en muestras de Singapur, una por el CDC (Urbani$S A R S$ ), y la última por el grupo de Canadá (Tor2).

La familia Coronaviridae incluye a los géneros $\mathrm{Co}$ ronavirus y Toravirus. Estos son virus ARN que poseen una cubierta y que causan enfermedades en humanos y en animales. Los Coronavirus tipos 229E y OC43 constituyen una de las principales causas de la gripe o catarro común en humanos y a veces ocasionan neumonías severas en ancianos, inmunocomprometidos y en neonatos. ${ }^{17-19}$ También se ha asociado a los Coronavirus con neumonías en reclutas militares. Filogenéticamente, la nueva variedad de Coronavirus identificada en pacientes con el SARS no se relaciona cercanamente con ninguna de las variedades previamente conocidas. ${ }^{19}$ Recientemente el doctor Gus Kousoulas, director de virología de la Escuela Veterinaria de la Universidad de Louisiana, ha sugerido que el SARS podría haberse transferido de ganado bovino hacia los humanos. Es- tas aseveraciones se basan en la descripción previa de un síndrome en ganado bovino denominado shipping disease o enfermedad de transporte, idéntico al SARS, e identificado en los años 90 del siglo pasado en ganado bovino del estado de Texas, en EUA. ${ }^{21}$

La identificación de la nueva variedad de Coronavirus como agente causal del SARS ha permitido el desarrollo de pruebas serológicas que ayudan a la identificación precisa de casos al desarrollar pruebas de laboratorio para estudiar posibles agentes antivirales con fines terapéuticos y, finalmente, para el desarrollo de una vacuna efectiva. ${ }^{17-19}$

Se ha sugerido que una posible combinación de los dos virus podría explicar la elevada tasa de ataque y la elevada tasa de letalidad en las series de casos canadienses. Asimismo, es posible que el Metaneumovirus humano sea un virus colonizador en pacientes con el SARS, pero ya que la tasa de colonización asintomática es desconocida, es difícil asumir hasta este momento de la epidemia que el virus refleja simplemente colonización de las vías aéreas. ${ }^{15,16} \mathrm{El}$ papel de la presencia del Metaneumovirus humano y de diversas especies de Chlamydia en algunos pacientes con el SARS requiere de estudios que incluyan grupos control para poder establecer causalidad o para determinar si estos agentes pueden ser cofactores de severidad clínica. ${ }^{8}$

\section{Mecanismo de transmisión}

Los casos del SARS se han asociado primordialmente a la transmisión a contactos cercanos (cara a cara). Existe evidencia de que algunos casos son particularmente infecciosos y a los cuales se ha denominado super-diseminadores. ${ }^{22-24}$ En Canadá los brotes se han caracterizado por afectar a trabajadores de la salud que han atendido a pacientes con el SARS, por ser contactos intradomicilarios y por la transmision intrafamiliar; en Taiwán la transmisión se ha limitado a contactos cercanos, sin afectar al personal de salud y en EUA la mayoría de los casos han ocurrido en personas que han viajado a zonas de alto riesgo y en sus contactos; y recientemente, en el personal de salud que ha atendido casos sospechosos. ${ }^{11,12}$

Resulta evidente que el riesgo de contraer el SARS es percibido en forma diferente por diversas organizaciones internacionales y por diversos países. Mientras que la OMS considera hasta el momento que la transmisión del SARS no es por vía respiratoria, el CDC ha mencionado repetidamente en sus reportes del Morbidity and Mortality Weekly Report, que la transmisión es por vía respiratoria. ${ }^{23-25}$

El mecanismo de transmisión no ha sido totalmente esclarecido, sin embargo, los reportes de contactos cercanos de pacientes con el SARS (contactos intra- 
domicilarios y personal de salud) sugieren que la diseminación ocurre a través de contacto o secreciones respiratorias. ${ }^{2,4,23-25}$ Asimismo, la cadena de transmisión que ocurrió en un hotel en Hong Kong, a través de un paciente que viajó de la provincia de Guandong a Hong Kong, es quizás la cadena de transmisión hasta el momento mejor descrita Esta sugiere que la transmisión ocurre a través de aerosoles respiratorios o por medio de fomites. Recientemente se ha implicado a los civetas, especie de gatos asiáticos, consumidos en el sur de China y considerados como cocina exótica, como posibles hospederos, debido a la identificación de cuatro cepas de virus similares al Coronavirus asociado al SARS en heces fecales de los civetas. ${ }^{10}$

Es a partir del caso índice en Hong Kong que se ha asociado, epidemiológicamente, la transmisión del SARS a otros países asiáticos y hacia otros continentes por huéspedes de este hotel. ${ }^{13-17}$ La descripción epidemiológica y clínica de los casos del SARS en Hong Kong, Canadá y EUA, proporciona una perspectiva clínica y epidemiológica de lo que ha sido descrito hasta el momento en la literatura en relación con el SARS.

\section{Brote del SARS en Hong Kong}

Las epidemias en Toronto y en Hong Kong se han caracterizado por involucrar principalmente a adultos relativamente jóvenes, con un promedio de edad de 39 a 42 años y, en ambos casos, el personal médico ha constituido la mayoría de los casos, $51 \%$ en Toronto, y de 28 a $50 \%$ en Hong Kong. ${ }^{26}$

El 15 de febrero de 2003 un paciente masculino de 64 años de edad viaja de la provincia de Guandong, en el sur de China, hacia Hong Kong a visitar a su familia. La cadena de transmisión y los casos se originan a partir de este caso índice, quien estuvo alojado como huésped en el Hotel Metropolitan en Hong Kong y, por otro lado, también da lugar a la cadena de transmisión en personal de salud que atendió a este paciente y a los contactos del hotel. ${ }^{5,13,17}$ Los casos secundarios identificados como contactos del caso inicial incluyeron cuatro trabajadores de la salud, dos de sus familiares y 12 pacientes que fueron huéspedes durante su estancia en el mismo hotel, y dos huéspedes que permanecieron después de su salida del hotel. El paciente, y uno de sus familiares murieron a los pocos días de insuficiencia respiratoria progresiva. Sin embargo, en investigaciones epidemiológicas subsecuentes, múltiples casos fueron asociados a este brote en Hong Kong. De uno de los huéspedes de ese hotel se originaron aproximadamente 34 casos en trabajadores de la salud y 37 contactos cercanos en Singapur. Eventos similares ocurrieron en Vietnam, Canadá, Irlanda y
EUA de pacientes que también se hospedaron en el mismo hotel. En forma similar, pacientes de este hotel fueron asociados, epidemiológicamente, con la diseminación de casos en diversos hospitales en Hong Kong, dando origen a múltiples casos en trabajadores de la salud (figura 1). Hasta principios de abril, Hong Kong es el segundo lugar después de China con el mayor número de casos y de muertes asociadas con el SARS. ${ }^{13}$

En el distrito de Kowloon, en Hong Kong, el edificio de departamentos de 33 pisos Amoy Gardens, ha sido también importante al proporcionar valiosa información epidemiológica sobre el posible mecanismo de transmisión de esta enfermedad. Hasta el momento, 131 casos del SARS han sido confirmados en residentes de este complejo de departamentos, y 241 residentes asintomáticos han estado en cuarentena, por 10 días, después del brote. El mecanismo de transmisión en este brote se ha asociado a posible contaminación del sistema de tuberías de agua con el Coronavirus. ${ }^{27}$

En la descripción clínica y epidemiológica de 10 casos del SARS, ocurridos en Hong Kong, el rango de edad varió de 35 a 72 años, siendo cinco de ellos mujeres y cinco hombres. Las manifestaciones clínicas en estos pacientes incluyeron: fiebre, disnea, tos no productiva, cefalea y escalofríos. ${ }^{13}$ En el examen físico se describió la presencia de estertores alveolares y matidez a la percusión. Linfopenia y elevación leve de transaminasas hepáticas fueron hallazgos de laboratorio. En todos los pacientes se encontraron infiltrados neumónicos a los rayos x del torax. Dos de los 10 pacientes descritos en esta serie de casos de Hong Kong,

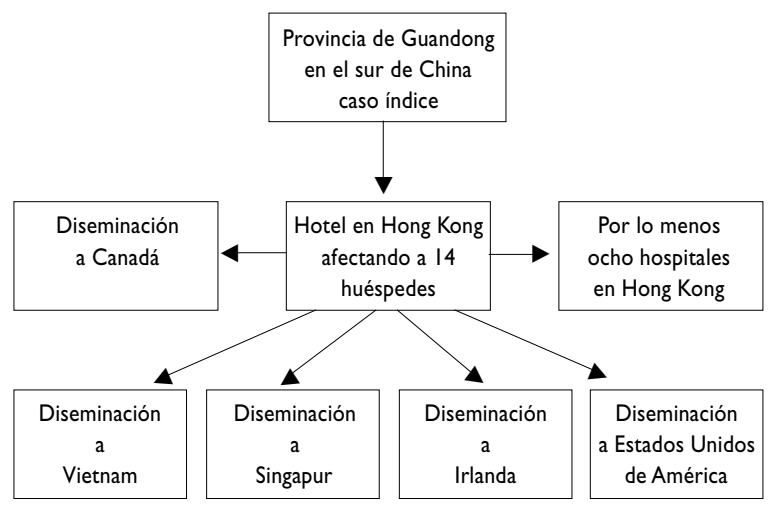

Figura I. Cadena de transmisión epidemiológica asociada a la diseminación de casos en Hong Kong Y HACIA OTROS CONTINENTES 
murieron y, en estudios de necropsia, se halló evidencia de hemorragia alveolar difusa. ${ }^{13}$

\section{Brote del SARS en Canadá}

En la primera serie de casos del SARS en Canadá, descritos en las ciudades de Toronto y Vancouver e identificados a principios de marzo, se encontró que $60 \%$ de los casos ocurrieron en hombres, y el rango de edad de los pacientes afectados fue de 24 a 78 años; ningún caso fue descrito en niños. La forma de transmisión en estos casos fue determinada por contacto cercano con casos del SARS. Los síntomas de presentación identificados incluyeron: fiebre, astenia, tos no productiva y disnea, síntomas asociados a la presencia, en placa de tórax, de infiltrados neumónicos en $100 \%$ de los casos. ${ }^{11}$ En estudios de laboratorio se demostró la presencia de linfopenia, elevación de la deshidrogenasa láctica, transaminasas hepáticas y de creatininfosfokinasa. Se requirió de intubación y ventilación mecánica por insuficiencia respiratoria progresiva en $50 \%$ de los pacientes. ${ }^{11-13}$ De esta serie original de 10 casos en Canadá, tres murieron (a la fecha han fallecido 26). En cinco pacientes se identificó la presencia de una variedad de Metaneumovirus humano en especímenes respiratorios de pacientes con el SARS y una variedad de Coronavirus fue encontrada en cinco pacientes; en cuatro pacientes se aislaron los dos patógenos. En la serie canadiense del SARS se reconoce una elevada morbilidad y mortalidad. ${ }^{11}$ A pesar del control inicial del brote en Toronto, se han identificado recientemente otros cinco casos a finales de mayo. ${ }^{10}$

\section{Brote del SARS en Estados Unidos de América}

Hasta el 30 de mayo, en 40 estados de EUA, se han reportado aproximadamente 355 casos del SARS al CDC. Estos números difieren de los de la OMS debido a la variación en la definición de casos. ${ }^{23-25}$ De estos números, 290 casos se han clasificado como sospechosos y 66 como casos probables. Aproximadamente 63 casos probables tenían como antecedente el haber viajado a regiones de alto riesgo 10 días antes de padecer los síntomas respiratorios y, de éstos, 33 (52\%) han viajado a China. ${ }^{28}$ Llama la atención que $81 \%$ de los casos han ocurrido en adultos, principalmente en el grupo de 18 a 64 años de edad. Sin embargo, la experiencia en EUA ha demostrado que el SARS también puede afectar a grupos durante la infancia. ${ }^{23-25,28}$

La mayoría de los pacientes reportados en EUA han presentado placas de tórax normales (53\%) y sólo $23 \%$ presentaron neumonía y por lo tanto cumplieron con los criterios de caso probable de la OMS. En cuanto a hospitalización, 40 han requerido estancia intra- hospitalaria por más de 24 horas y únicamente dos pacientes han requerido de ventilación mecánica asistida por insuficiencia respiratoria progresiva. A la fecha, en EUA no se ha reportado ninguna muerte asociada al SARS. La gravedad de la enfermedad y la tasa de ataque en pacientes diagnosticados en EUA ha sido menos grave que en otras áreas, como en Hong Kong. ${ }^{23-25}$

Los CDC y la OMS han recomendado no viajar a Hong Kong o a la provincia de Guandong, en China, Hanoi, en Vietnam y Singapur, aunque a finales de abril se levantó la cuarentena oficial a Vietnam, Toronto y a Singapur; a fines de mayo la recrudescencia de nuevos casos en Toronto es preocupante. Las recomendaciones generales incluyen monitorizar la presencia de síntomas asociados a este síndrome y acudir a revisión médica si ocurre la aparición de estos síntomas dentro de los 10 días después del viaje a estas zonas. ${ }^{11-13,23-25}$

Adicionalmente, se recomienda la aplicación de medidas de control nosocomial en aquellas personas en quienes se sospecha la presencia del SARS. En forma similar, en individuos en quienes se sospecha clínicamente el SARS y son enviados a casa, se recomienda el aislamiento intradomiciliario con limitación de actividades (no asistir al trabajo, escuela o áreas públicas) hasta 10 días después de la mejoría de la fiebre y de los síntomas respiratorios. ${ }^{5,13}$

\section{Características clínicas y diagnóstico}

Resulta evidente, en la información clínica descrita en los diversos reportes hasta el momento publicados por diversos países, que el periodo de incubación del SARS es típicamente de 1 a 10 días, con un promedio de cinco. Mientras más largo es el periodo de incubación más se facilita la transmisión a casos secundarios antes de la aparición de síntomas. ${ }^{9-14}$ La enfermedad se inicia, generalmente, con un periodo prodrómico de fiebre mayor a $38{ }^{\circ} \mathrm{C}$. La fiebre es frecuentemente elevada, en ocasiones asociada con escalofríos y se puede acompañar de cefalea, astenia y mialgias. Algunas personas presentan síntomas respiratorios al inicio de la enfermedad. Algunos pacientes pueden presentar diarrea durante el periodo prodrómico. Después de 3 a 7 días, comienza una fase de síntomas respiratorios con tos seca no productiva y disnea, que puede progresar a disnea severa con hipoxemia (cuadro II). En ocasiones la presencia de insuficiencia respiratoria asociada con la neumonía atípica requiere de intubación endotraqueal con ventilación mecánica en 10 a $20 \%$ de los casos. ${ }^{11,12}$ En tres series clínicas diferentes, de 6 a $20 \%$ de los pacientes presentan atípicamente diarrea, lo cual puede asociarse a una mayor transmisibilidad.

salud pública de méxico / vol.45, no.3, mayo-junio de 2003 
La radiografía de tórax puede ser normal durante el periodo prodrómico e inclusive durante el curso clínico de la enfermedad. En la mayoría de los pacientes la fase respiratoria coincide con infiltrados focales intersticiales que evolucionan a infiltrados intersticiales generalizados. ${ }^{11,12,17,29}$ En fases tardías pueden encontrarse áreas de consolidación alveolar (cuadro II).

En estudios de laboratorio, en fases tempranas, se puede encontrar una disminución significativa en la cuenta total de linfocitos, mientras que la cuenta total leucocitaria es normal o se encuentra disminuida. Coincidiendo con el pico de la fase de síntomas respiratorios, aproximadamente $50 \%$ de los pacientes desarrollan leucopenia y trombocitopenia (plaquetas entre 50000 y 150 000/ 1. Existe también elevación de los niveles de creatinin-fosfokinasa y de transaminasas hepáticas (dos a seis veces del límite superior normal). ${ }^{9-14}$

El algoritmo diagnóstico utilizado en casos que pudieran sugerir el SARS debe incluir radiografía de tórax, oximetría de pulso, hemocultivos, tinción de gram de muestras de esputo, pruebas diagnósticas para patógenos virales (Influenza A, Virus sincicial respiratorio). Además, especímenes clínicos (respiratorios, sangre y suero) deben enviarse a laboratorios de referencia como el del Instituto Nacional de Diagnóstico y Referencia Epidemiológicos, en México, y otros como los CDC, en Atlanta GA, EUA, para ser evaluados y confirmar el diagnóstico. ${ }^{25}$ Las instrucciones para la recolección y empaquetamiento de muestras para envío al CDC se encuentran disponibles en el siguiente sitio: http:/ / www.cdc.gov/ncidod/sars/intspecimens.htm.

\section{Cuadro II \\ Características clínicas, radiográficas Y DE LABORATORIO EN PACIENTES CON EL SÍNDROME AGUDO RESPIRATORIO SEVERO}

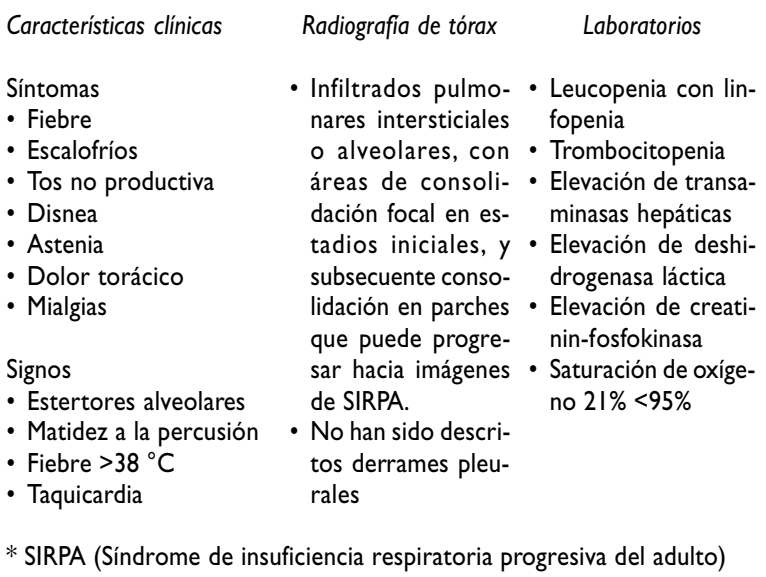

A la fecha se cuenta con ensayos serológicos y de diagnóstico molecular (PCR) para la confirmación diagnóstica en muestras de pacientes con el SARS. Las pruebas de PCR pueden detectar la presencia del $\mathrm{Co}^{-}$ ronavirus en diversos especímenes clínicos (sangre, heces fecales, secreciones respiratorias y otras secreciones corporales). Las secuencias genómicas para la realización de la prueba de PCR se encuentran disponibles a través de la red de laboratorios de la OMS. Sin embargo, kits diagnósticos con controles positivos y negativos están también comercialmente disponibles. La técnica de PCR para la detección del Coronavirus es poco sensible pero muy específica, por lo cual una prueba negativa no descarta la posibilidad de la presencia del virus en muestras clínicas.

Las pruebas serológicas disponibles para la identificación de anticuerpos IgM e IgG producidos en respuesta a la infección por Coronavirus se realizan a través del método de ELISA (Enzyme Linked ImmunoSorbant Assay) y por pruebas de inmunofluorescencia que detectan IgM en suero después de diez días de la enfermedad. Finalmente, el cultivo celular está siendo realizado en los laboratorios de la red de referencia de la OMS. ${ }^{10}$

La evolución clínica del síndrome es variable y puede manifestarse como una forma leve o severa. Muchos pacientes requieren ser ingresados a las unidades de terapia intensiva, tal como sucedió en $21 \%$ de los casos en Toronto, y en 38\% de los de Hong Kong; además, requirieron asistencia mecánica ventilatoria $14 \%$ de los casos de Toronto y entre 14 a 38\% de los de Hong Kong. ${ }^{26}$

La tasa de letalidad al principio de la epidemia se consideró únicamente de 3 a $4 \%$. Sin embargo, hasta el 21 de mayo, la tasa de fatalidad global ha ido incrementándose hasta llegar a una tasa global de $5.6 \%{ }^{10}$ No obstante, esta tasa ha variado desde $3 \%$ hasta $16 \%$, dependiendo de la región afectada. Taiwán, Hong Kong y Toronto han presentado las mayores tasas de letalidad con $12 \%, 14.8 \%$, y $16 \%$, respectivamente. Asimismo, un modelo matemático reciente ha sugerido que, tomando en cuenta determinantes epidemiológicas de la transmisión del Coronavirus, la letalidad se ha asociado a diversos cofactores, principalmente la edad, la cual es quizás, hasta el momento, el factor determinante más importante asociado a una mayor tasa de letalidad (13.2\% en pacientes menores de 60 años y de $43.3 \%$ en personas mayores de 60 años, asumiendo una distribución paramétrica). ${ }^{30}$ Se ha observado que el SARS no solamente ocurre rara vez durante la infancia, sino que llega a manifestarse clínicamente con cuadros clínicos menos agresivos que en adultos. ${ }^{31} \mathrm{Al}-$ gunos de los contactos cercanos a casos del SARS desarrollan fiebre sin síntomas respiratorios, sugiriendo 
que en muchos de los casos la enfermedad no progresa hacia la fase de síntomas respiratorios. ${ }^{11-13}$ En una serie de casos de Hong Kong, recientemente publicada, los factores de riesgo asociados a mayor morbimortalidad fueron la presencia de edad mayor de 50 años, linfopenia y elevación de transaminasas. ${ }^{8,19}$

Los criterios de los CDC y de la OMS para la definición de casos sospechosos, la cual se considera preliminar y no muy específica, incluye los siguientes aspectos:

- $\quad$ Temperatura $>38^{\circ} \mathrm{C}$

- Uno o más de los siguientes síntomas y signos respiratorios ( tos, disnea, hipoxia, radiografía de tórax con hallazgos característicos de neumonía o SIRPA)

- Historia de viaje a un área considerada como de riesgo, ${ }^{*}$ con casos sospechosos o confirmados del SARS o con cadenas de transmisión local (excluyendo casos en áreas de casos secundarios, limitados a trabajadores de la salud o a contactos cercanos de casos) dentro de 10 días desde la aparición de síntomas, o un contacto cercano dentro de un marco de 10 días de la aparición de síntomas, con un caso del SARS, con una persona con síntomas respiratorios que haya viajado a zonas de alto riesgo o de casos sospechosos del SARS.

\section{Tratamiento}

El tratamiento empírico ha incluido diversos esquemas con antibióticos de amplio espectro, para cubrir agentes que ocasionan neumonías bacterianas típicas y atípicas. En muchas ocasiones se han utilizado, en forma empírica, agentes antivirales inhibidores de la neuraminidasa como el oseltamivir y otros como la ribavirina, asociados a esteroides orales o intravenosos. ${ }^{11-13,17}$

La eficacia de estos tratamientos empíricos en las series de Canadá y Hong Kong no se ha establecido con precisión. Hasta el momento no existe un tratamiento específico para este síndrome. Sin embargo, Peiris y colaboradores sugieren, con base en su serie de 50 pacientes en Hong Kong, que la utilización de ribavirina y glucocorticoides intravenosos demostra-

\footnotetext{
* Hong Kong, República Popular de China, Taiwán.

Nota: La OMS considera los casos sospechosos como casos probables, cuando se cumplen uno de los siguientes tres criterios: (a) caso sospechoso con hallazgos radiográficos de neumonía o del SIRPA; (b) caso sospechoso con evidencia del SIRPA en necropsia, y (c) caso sospechoso con una o más pruebas de laboratorio positivas para la presencia del Coronavirus.
}

ron cierta eficacia clínica. ${ }^{19}$ Sin embargo, debido a la falta de evidencia clínica y de laboratorio en diversas series, además de una elevada toxicidad (en $76 \%$ de los casos) las autoridades canadienses no están recomendando ni autorizando el empleo de ribavirina. ${ }^{29}$ No obstante la ausencia de controles en el estudio de Peiris y colaboradores, ${ }^{19}$ y ante la, hasta este momento, imposibilidad de desarrollar ensayos clínicos controlados sugerimos que, cuando se decida utilizar empíricamente estos medicamentos, se documente cuidadosamente la experiencia clínica para poder trazar conclusiones más específicas acerca de la utilización clínica de dichos fármacos.

El tratamiento es fundamentalmente de apoyo hemodinámico, ventilatorio y de cuidados intensivos cuando esto se requiera. ${ }^{11-13,17}$ Reportes recientes en el modelo animal de infección por el Coronavirus causante de hepatitis en ratones sugieren que el bloqueo de la protein-kinasa activadora de mitosis p38, podría resultar un sitio de bloqueo molecular ideal en el desarrollo de antivirales efectivos contra el Coronavirus causante del SARS. ${ }^{32}$ El hallazgo molecular de que los salicilatos pueden inducir la activación de la MAP-kinasa p38 es evidencia suficiente para no recomendar la utilización de estas sustancias en pacientes con el SARS. ${ }^{33}$ Con base en lo anterior, tambien consideramos que no obstante no existir ninguna evidencia clínica de la posible asociación entre el uso de salicilatos en pacientes con el SARS y un desenlace más severo, sería conveniente evitar el uso de salicilatos en estos pacientes.

El hallazgo del bloqueo de la replicación viral a traves del fármaco AG7088, por medio de reprimir a la proteinasa principal del virus (3Clpro), ofrece quizás una esperanza del posible desarrollo cercano de antivirales efectivos contra el Coronavirus. ${ }^{34}$ Se ha descrito recientemente que en estudios de replicación in vitro el interferón alfa resulta también altamente efectivo, lo cual no ha sido hasta el momento probado clínicamente. $^{29}$

El papel de los esteroides en el tratamiento de esta enfermedad no está bien esclarecido. Sin embargo, su uso en diversas series se ha asociado a un mejor pronóstico, especialmente cuando han sido utilizados entre los días 6 a 8 de la enfermedad. Nichols et a ${ }^{\beta 5}$ realizaron estudios postmortem en seis pacientes que fallecieron debido al SARS, y encontraron proliferación hiperplásica epitelial asociada con hemofagocitosis. La presencia de este fenómeno se ha asociado clínicamente a una regulación alterada de la cascada inflamatoria de las citocinas, lo cual podría considerarse como un marcador que sugiere cómo la utilización clínica de esteroides podría ser de gran importancia. ${ }^{35}$ 


\section{Medidas de prevención y control del SARS}

El CDC y la OMS recomiendan que para todo contacto con un paciente sospechoso de tener el SARS es fundamental el lavado estricto de manos con agua y jabón o, alternativamente, con soluciones de alcohol. . $^{36-38}$

Si la persona con sospecha de tener el SARS es ingresada al hospital, el personal encargado de epidemiología hospitalaria deberá ser notificado inmediatamente. Las medidas de prevención intrahospitalaria incluyen: ${ }^{38}$

- Precauciones estándares (por ejemplo, lavado de manos); adicionalmente, el personal de salud debe utilizar lentes de protección

- Precauciones de contacto (por ejemplo, la utilización de batas desechables y guantes)

- Precauciones respiratorias, cuartos de aislamiento respiratorio y la utilización de máscaras protectoras N-95. Si las medidas respiratorias no pueden aplicarse con estos estándares, los pacientes deben de colocarse en cuartos individuales no compartidos, y todas las personas que entren al cuarto deben utilizar cubreboca quirúrgico. En casos de procedimientos de alto riesgo, como son aquellos generadores de aerosol, como broncoscopía e intubación endotraqueal, las autoridades de salud pública de Canadá han recomendado la utilización de doble guante y máscara respiratoria e inclusive doble protección conjuntival. ${ }^{39}$

Los casos sospechosos de tener el SARS que se encuentren médicamente estables y que son enviados a su domicilio deberán ser instruidos en la utilización de cubreboca quirúrgico para evitar contagios intra domiciliarios. ${ }^{37-40}$ Las personas que comparten el mismo domicilio deben lavarse las manos frecuentemente. Información adicional sobre el tema y sobre lineamientos de reporte y manejo de caso sospechoso en todo el territorio nacional se puede consultar en el portal de la Dirección General de Epidemiología, de la Secretaría de Salud: http://www.epi.org.mx.

El manejo de las personas que han sido expuestas a casos sospechosos del SARS debe hacerse como lo indica el siguiente algoritmo (figura 2), sugerido por el CDC y la OMS.

\section{Conclusiones}

El SARS representa uno de los mejores ejemplos descritos hasta el momento de una infección emergente en la que se demuestra que una infección puede viajar de un continente a otro en plazo de días o semanas.

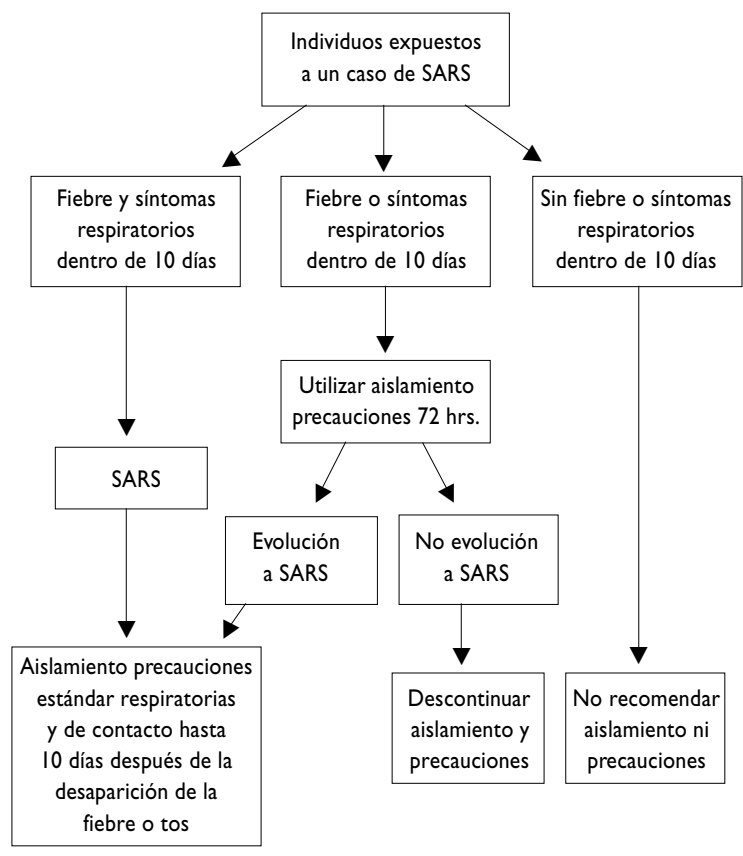

Tomado de: CDC y OMS

(http://www.cdc.gov/ncidod/sars/exposuremanagementframe.htm) (Referencias ${ }^{37-40}$ )

Figura 2. Algoritmo de manejo en caso de exposición A UN CASO SOSPECHOSO DEL SARS

Con el advenimiento de la epidemia del SARS se ha comprobado que la colaboración internacional es fundamental en la respuesta a epidemias ocasionadas por infecciones emergentes. La respuesta internacional generada por el SARS es un evento sin precedentes en la historia de la salud pública mundial, puesto que 11 laboratorios, alrededor del mundo, trabajan hacia un solo fin, el intercambio libre de la información que permita el control de esta epidemia.

\section{Referencias}

I. Severe acute respiratory syndrome (SARS).Wkly Epidemiol Rec 2003;78:8I-83.

2. Drazen JM. Case clusters of the severe acute respiratory syndrome. N Engl J Med 2003;348(20):6-7; versión electrónica (marzo 31 de 2003). Disponible en: http://www.nejm.com(10.1056/NEJMe030062).

3. CDC Update: Outbreak of severe acute respiratory syndromeWorldwide, 2003. MMWR Morb Mortal Wkly Rep 2003;52(I3):269-272. 4. Gerberding JL. Faster...but fast enough? Responding to the epidemic of severe acute respiratory syndrome. N Engl J Med 2003;348(20):2030203I; versión electrónica (abril I 2 de 2003). Disponible en: http:// www.nejm.com(10.1056/NEJMe030067). 
5. CDC Update: Outbreak of severe acute respiratory syndromeWorldwide, 2003. MMWR Morb Mortal Wkly Rep 2003;529(I2): 24I-248.

6. News Lancet. Beijing doctor alleges SARS cases cover-up in China. Lancet 2003;36I(9366): 1357.

7. Kahn J. China discovers medical secrecy is expensive. New York Times 2003 April 17.

8. Falsey AR, Walsh EE. Novel Coronavirus and severe acute respiratory syndrome. Lancet 2003;36(9366):13 |2-13 |3; versión electrónica (abril 8 de 2003). Disponible en: http://image.thelancet.com/extras $/ 03 \mathrm{~cm}+8 /$ neb.port.

9. World Health Organization. Cumulative number of reported cases of severe acute respiratory syndrome (3 de abril de 2003). Disponible en: http://www.who.int/esr/sarscountry/2003_04_03/en/. 10. World Health Organization. Severe acute respiratory syndrome Homepage. Disponible en: http://www.who.int/csr/sars/en/

II. Poutanen SM, Low DE, Henry B, Finkelstein S, Rose D, Green K et al. Identification of severe acute respiratory syndrome in Canada. N Engl J Med 2003;348(20): 1995-2005; versión electrónica (marzo 31 de 2003). Disponible en: http://www.nejm.com(I 0.1056/NEJMoa030634). 12. Tsang KW, Ho PL, Ooi GC, Yee WK, Wang T, Chang-Yeung M et al. A cluster of cases of severe acute respiratory syndrome in Hong Kong. $\mathrm{N}$ Engl J Med 2003;348(20): 1977-1985; versión electrónica (marzo 3I de 2003). Disponible en: http://www.nejm.org(I0.1056/NEJMoa030666). 13. CDC. Preliminary clinical description of severe acute respiratory syndrome. MMWR Morb Mortal Wkly Rep 2003;52(I2):255-256. 14. Pearson H. Mystery virus slow to yield its identity as patient numbers rise. Nature 2003;422(364):423-424; versión electrónica, (marzo 27 de 2003). Disponible en: http://www.nature.com(doi. 10.1038/ 42324).

15. Bolvin G,AbedY, Pelletier G.Virological features and clinical manifestations associated with human metaneumovirus: $A$ new Paramyxovirus responsible for acute respiratory-tract infections in all age groups.J Infect Dis 2002;186:1330-1334.

16. Stockton J, Stephenson I, Fleming D, Zambon M. Human metapneumovirus as a cause of community-acquired respiratory illness. Emerg Infect Dis 2002;8(9):84.

17. Ksiazek TG, Erdman D, Goldsmith C, Zaki SR, Peret T, Emery S et al. A novel Coronavirus associated with severe acute respiratory syndrome. N Engl J Med 2003;348(20):1953-1966; versión electrónica (abril 10 de 2003). Disponible en: http://www.nejm.com(I 0.1056/NEJMoa03078I).

18. Drosten C, Gunther C, Preiser W, van der Werf S, Hans-Reinhard B, Becker $S$ et al. Identification of a novel Coronavirus in patients with severe acute respiratory syndrome. N Engl J Med 2003;348(20):1967. 1976; versión electrónica, (abril 16 de 2003). Disponible en:http:// www.nejm.com(I 0.1056/NEJMoa030747)/NEJMoa030747v2.pdf). 19. Peiris JSM, Lai ST, Poon LLM, Guan Y,Yam LYC, Lim W et al. Coronavirus as a possible cause of severe acute respiratory syndrome. Lancet 2003;361:1319-1325; versión electrónica (abril 8 de 2003). Disponible en: http://image.thelancet.com/extras/03art3477web.pdf. 20. Fouchier RA, Kuiken T, Schutten M,Van Amerongen G,Van Doornum G], Bernadette $G$ et al.Aetiology: Koch's postulates fulfilled for SARS virus. Nature 2003; 423(240): 333-340; versión electrónica (mayo 15 de 2003). Disponible en: http://www.nature.com(doi: $10.1038 / 423240^{\mathrm{a}}$ ).

21. NaaNes M. U.S. Immunization News. LSU Professor hopes to develop vaccine against SARS. Baton Rouge Advocate. Versión electrónica (abril 24 de 2003). Disponible en: http:// www.theadvocate.com.

22.WHO. Severe Acute Respiratory Syndrome (SARS)-multi-country outbreak-Update 30. Status of diagnostic tests, significance of "super spreaders", situation in China.Versión electrónica (abril 15, 2003). Disponible en: http://www.who.int/csr/don2003_04_I5/en/.
23. CDC. Update. Severe acute respiratory syndrome. United States. 2003. MMWR Morb Mortal Wkly Rep 2003; 52 (I5):332-336.

24. News Lancet. Global update on SARS cases. Lancet 2003;36I(9366): I289.

25. CDC. Diagnosis/Evaluation of severe acute respiratory syndrome (SARS). CDC website: http://www.cdc.gov/ncidod/sars/diagnosis.htm. 26. Masur $\mathrm{H}$, Ezequiel $\mathrm{E}$, Lane $\mathrm{CH}$. Severe acute respiratory syndrome. Providing care in the face of uncertainty. JAMA 2003;289(2I):10-12. 27. Cyranoski D,Abbot A. Apartment complex holds clues to pandemic potential of SARS. Nature 2003;3-4; versión electrónica (mayo I de 2003). Disponible en: http://www.nature.com(doi:10.1038/423003a). 28. CDC. Update. Severe acute respiratory syndrome: United States, May 2I, 2003;52(20):466-468.

29. Booth CM, Matukas LM, Tomlinson GA, Rachlis AR, Rose DB, Dwosh $\mathrm{HA}$ et al. Clinical features and short-term outcomes of I44 patients with SARS in the greater Toronto area. JAMA 2003;289(2I):I-8.

30. Donnelly CA, Ghani AC, Leung GM, Hedley AJ, Fraser C, Riley $S$ et al. Epidemiological determinants of spread of causal agent of severe acute respiratory syndrome in Hong Kong. Lancet 2003;361:1761-1766; version electrónica (mayo 17 de 2003). Disponible en: (http:// image.thelancet.com/extras/03art4453web.pdf).

31. Hon KL, Leung CW, Cheng WT, Chan PK, Chu WC, Kwan YW et al. Clinical presentations and outcome of severe acute respiratory syndrome in children. Lancet 2003;361:1701-1703; version electrónica (abril 29 de 2003). Disponible en: http://image.thelancet.com/extras/ 03let 4 I27web.pdf.

32. Ordan O, Rotem R, Jaspers I, Fiescher E. Stress-responsive JNK mitogen-activated protein kinase mediates aspirin-induced suppression of BI6 melanoma acellular proliferation. Br J Pharmacol 2003:138: II56-1162.

33. McGilvray ID, Lu Z, Dackiw AP, Marshall JC, Kapus A, et al. Murine hepatitis virus strain- 3 induces the macrophage prothrombinase fgl-2 through p38 mitogen-activated protein-kinase activation.J Biol Chem 1998;273(48): II I56-I I62.

34. Anand KZ,Washwani P, Mesters JR, Hilgenfeld R. Coronavirus mainproteinase (3Clpro) structure: Basis for design of anti-SARS drugs. Science 2003; versión electrónica ( 13 de mayo de 2003). Disponible en: http://www.sciencemag.org(doi.I I 26/science. 1084658).

35. Nicholss JM, Poon LLM, Lu KC, Wai FN, Loung CY, Hui PK et al. Lung pathology of fatal severe acute respiratory syndrome. Lancet 2003;361:1773-1778; versión electrónica (I6 de mayo de 2003). Disponible en: http://image.thelancet.com/extras/03art4347web.pdf. 36. HoW. Guidelines on management of severe acute respiratory syndrome (SARS). Lancet 2003;361:1313-13|4; versión electrónica (8 de abril de 2003). Disponible en: http://image.thelancet.com/extras/ $03 \mathrm{cmt} 89$ web.pdf.

37. Sing Tao LIT, Buckley TA, Yap FHY, Sung JJY, Joynt GM. Severe acute respiratory syndrome (SARS) Infection Control. Lancet 2003;36I: |386- I387.

38. CDC. Updated interim domestic infection control guidance in the health care and community settings for patients with suspected SARS. Versión electrónica (April 25, 2003). Disponible en: http://www.cdc.gov/ sars.

39. Health Canada. Cluster of severe acute respiratory syndrome cases among protected health care workers - Toronto, April 2003. Canada Communicable Disease Report; versión electronica (18 de abril de 2003). Disponible en: http://www.hc-sc.gc.ca/pphb-dgspsp/publicat/ccdrrmtc/03vol29/prev/dr-sars0515.html.

40. Lee N, Hui D, Wu A, Chan P, Cameron P, Joynt GM et al. A major outbreak of SARS in Hong Kong. N Engl J Med 2003;348(20): 1986-1994; versión electrónica (16 de abril de 2003). Disponible en: http:// www.nejm.com(I0.1056)/NEJMoa030685). 